\title{
Controle alternativo de podridões pós-colheita de framboesas
}

\author{
Lucimara Rogéria Antoniolli(1), Gildo Almeida da Silva(1), Silvio André Meirelles Alves ${ }^{(2)}$ e Laís Moro(3)
}

\begin{abstract}
(1)EmbrapaUvaeVinho, CaixaPostal130,CEP95700-000BentoGonçalves, RS.E-mail:lucimara@cnpuv.embrapa.br, gildo@cnpuv.embrapa.br (2)Embrapa Uva e Vinho, Estação Experimental de Fruticultura de Clima Temperado, Caixa Postal 1513, CEP 95200-000 Vacaria, RS. E-mail: silvio@cnpuv.embrapa.br (3)Universidade de São Paulo, Faculdade de Ciências Farmacêuticas, Caixa Postal 66083, CEP 05508-000 São Paulo, SP. E-mail: lais.moro@gmail.com
\end{abstract}

Resumo - O objetivo deste trabalho foi avaliar o efeito de tratamentos pré-colheita sobre a ocorrência de podridões pós-colheita e sobre os atributos de qualidade de framboesas (Rubus idaeus L.) 'Heritage'. As frutas foram pulverizadas com um dos seguintes tratamentos: água destilada (controle), $6 \mathrm{~g} \mathrm{~L}^{-1}$ de quitosana, $100 \mathrm{mg} \mathrm{L}^{-1}$ de dióxido de cloro, Bacillus amyloliquefaciens, Curtobacterium pusillum ou Saccharomyces cerevisiae. Foram realizadas colheitas aos 3, 7 e 14 dias após a aplicação dos tratamentos. Após cada uma das colheitas, realizadas no estádio de maturação comercial (coloração rosa), as frutas foram inoculadas individualmente com suspensão de conídios ( $2 \times 10^{5}$ conídios $\left.\mathrm{mL}^{-1}\right)$ de Botrytis cinerea ou Rhizopus stolonifer. As frutas foram mantidas a $12 \pm 0,5^{\circ} \mathrm{C}$ por sete dias e avaliadas quanto à incidência de podridões e quanto aos principais atributos de qualidade. Bacillus amyloliquefaciens, $C$. pusillum e $S$. cerevisiae proporcionaram menor área abaixo da curva de progresso da incidência das podridões por Botrytis e Rhizopus. Os agentes de controle biológico avaliados não interferem negativamente sobre os atributos de qualidade das frutas, e, portanto, são alternativas potenciais no controle de podridões pós-colheita de framboesas.

Termos para indexação: Bacillus amyloliquefaciens, Curtobacterium pusillum, Rubus idaeus L., Saccharomyces cerevisiae, controle biológico, pequenas frutas, quitosana.

\section{Alternative control of raspberry postharvest decay}

Abstract - The objective of this work was to evaluate the effect of pre-harvest treatments on post-harvest decay and on quality of 'Heritage' raspberries (Rubus idaeus L.). Fruit were sprayed with one of the following treatments: distilled water (control), $6 \mathrm{~g} \mathrm{~L}^{-1}$ of chitosan, $100 \mathrm{mg} \mathrm{L}^{-1}$ of chlorine dioxide solution, Bacillus amyloliquefaciens, Curtobacterium pusillum or Saccharomyces cerevisiae suspensions. Harvest was done at 3 , 7, and 14 days after spray treatment. After each harvest, at commercial maturity (when fruit were pink), fruit were individually inoculated with conidial suspension $\left(2 \times 10^{5}\right.$ conidia $\left.\mathrm{mL}^{-1}\right)$ of Botrytis cinerea or Rhizopus stolonifer. Fruit were kept at $12 \pm 0.5^{\circ} \mathrm{C}$ for seven days and were evaluated for decay and main quality attributes. Bacillus amyloliquefaciens, C. pusillum, and S. cerevisiae provided smaller area under disease progress curve of decays caused by Botrytis and Rhizopus. The biological control agents evaluated do not adversely affect fruit quality attributes, and, therefore, are potential alternatives to control post-harvest decay of raspberries.

Index terms: Bacillus amyloliquefaciens, Curtobacterium pusillum, Rubus idaeus L., Saccharomyces cerevisiae, biological control, berries, chitosan.

\section{Introdução}

As pequenas frutas são muito apreciadas por sua coloração, sabor, aroma e textura, e, mais recentemente, têm sido valorizadas pelos benefícios proporcionados à saúde, decorrentes dos elevados teores de antioxidantes, vitaminas, minerais, fibras, ácido fólico, entre outros (Zhao, 2007). As framboesas (Rubus idaeus L.) destacam-se por sua coloração e seu sabor; entretanto, são altamente perecíveis e apresentam alterações poucas horas após a colheita (Nunes, 2008).
A deterioração causada por patógenos é a principal causa de perdas em pós-colheita de pequenas frutas, em virtude da fragilidade de sua epiderme. Em framboesas, especificamente, a abertura da cavidade do receptáculo, após a colheita, facilita a contaminação por esses agentes. O mofo-cinzento, causado por Botrytis cinerea, e a podridão de Rhizopus, causada por Rhizopus stolonifer, são as doenças fúngicas mais comuns em pequenas frutas durante o armazenamento (Bower, 2007; Mitcham, 2007).

A Instrução Normativa Conjunta (Brasil, 2010), que trata das culturas com suporte fitossanitário 
insuficiente, permite a extrapolação do limite máximo de resíduo (LMR) de uma cultura representativa de um subgrupo para as demais culturas do mesmo subgrupo. Assim, os fungicidas registrados no Ministério da Agricultura, Pecuária e Abastecimento (MAPA) para o controle do mofo-cinzento em morangos (Agrofit, 2011) também podem ser utilizados em framboesas. No entanto, a tendência atual é a substituição dos agrotóxicos convencionais por tratamentos alternativos que aliem segurança alimentar com sustentabilidade da agricultura (Bower, 2007).

A quitosana é um polímero natural de alto peso molecular, atóxico e biodegradável. É obtida por meio da desacetilação alcalina da quitina, que é encontrada naturalmente nas paredes celulares de alguns fungos e compõe a maior parte do exoesqueleto de insetos, crustáceos e invertebrados marinhos (Pen \& Jiang, 2003; Devlieghere et al., 2004; Han et al., 2005; Mazaro et al., 2008). A comprovada atividade fungistática desse polímero o torna uma alternativa potencial no controle de podridões pós-colheita de frutas. De acordo com El Ghaouth et al. (1991), a quitosana foi tão eficiente quanto o fungicida Rovral no controle de podridões pós-colheita de morangos. Reddy et al. (2000) verificaram que a pulverização pré-colheita de quitosana é eficiente para controlar $B$. cinerea em morangos e manter a qualidade das frutas, em níveis aceitáveis, por até quatro semanas de armazenamento a $3^{\circ} \mathrm{C}$

O dióxido de cloro é uma alternativa ao uso dos produtos clorados, com reconhecida eficiência na sanitização de frutas e na redução de podridões causadas por $B$. cinerea, Penicillium expansum e $R$. stolonifer (Zoffoli et al., 2005). Segundo Carvalho et al. (2009), o tratamento com dióxido de cloro a $0,1 \%$ reduziu a incidência de Rhizopus spp. em pêssegos. Para o tratamento profilático de morangos, a recomendação do fabricante é de três aplicações $\left(50 \mathrm{mg} \mathrm{L}^{-1}\right)$ antes da colheita.

A utilização de microrganismos antagônicos também se destaca como estratégia no controle das podridões pós-colheita de frutas. As bactérias Bacillus amyloliquefaciens e Curtobacterium pusillum são eficientes no controle de fungos fitopatogênicos, como B. cinerea. Mari et al. (1996) observaram que a ocorrência de podridões foi reduzida em tomates tratados com B. amyloliquefaciens e inoculados artificialmente com $B$. cinerea durante sete dias de armazenamento a $20^{\circ} \mathrm{C}$. De acordo com Piccinin et al. (2005), a levedura Saccharomyces cerevisiae apresenta potencial para o controle de doenças de plantas, uma vez que apresenta capacidade de sintetizar antibióticos e habilidade em competir por espaço e nutrientes. Contudo, pouco se sabe quanto a sua ação sobre as podridões pós-colheita de frutas.

O objetivo deste trabalho foi avaliar o efeito de tratamentos pré-colheita sobre a ocorrência de podridões pós-colheita e sobre os atributos de qualidade de framboesas 'Heritage'.

\section{Material e Métodos}

Framboesas 'Heritage' cultivadas em área comercial no Município de Vacaria, RS $\left(28^{\circ} 30^{\prime} \mathrm{S}\right.$ e $50^{\circ} 56^{\prime} \mathrm{W}$, a $971 \mathrm{~m}$ de altitude), foram pulverizadas com um dos seguintes tratamentos: solução de $6 \mathrm{~g} \mathrm{~L}^{-1}$ de quitosana purificada, conforme Reddy et al. (2000); $100 \mathrm{mg} \mathrm{L}^{-1}$ de dióxido de cloro (Tecsa Clor a 5\%); ou suspensão de B. amyloliquefaciens, C. pusillum ou S. cerevisiae, contendo $10^{7}$ células por $\mathrm{mL}$. Cada microrganismo foi cultivado em condição ambiente $\left(25^{\circ} \mathrm{C}\right)$ e aeróbica (2 vvm, volume de ar por volume de meio por min), em recipientes de vidro (Pirex) contendo $15 \mathrm{~L}$ de meio líquido $\mathrm{G} 7 \mathrm{e} 10 \mathrm{~g} \mathrm{~L}^{-1}$ de sacarose, de acordo com Silva \& Almeida (2006). As plantas controle foram pulverizadas com água destilada.

Antes da aplicação dos tratamentos, foi realizada a colheita das frutas em estádios de maturação para consumo in natura e processamento, apresentando coloração rosa, vermelha e vermelha escura. Assim, todas as frutas em estádios de maturação anteriores a esses permaneceram nas plantas e receberam a aplicação dos tratamentos. A pulverização, realizada uma única vez com pulverizador manual, foi direcionada às frutas até o ponto de escorrimento. Foram pulverizados $5 \mathrm{~L}$ de cada tratamento para aproximadamente 100 plantas dispostas em fila, com espaçamento adensado. Foram realizadas três colheitas consecutivas em intervalos de 3, 7 e 14 dias após a aplicação dos tratamentos. A incidência de podridão foi determinada com base no valor médio de incidência nos frutos das três colheitas, após 3 e 7 dias de incubação (pós-colheita).

As frutas foram colhidas no estádio de maturação para consumo in natura (coloração rosa), acondicionadas em recipientes de polietileno tereftalato (PET), para evitar a ocorrência de danos mecânicos, e imediatamente 
transportadas ao Laboratório de Fitopatologia da Estação Experimental de Fruticultura de Clima Temperado da Embrapa Uva e Vinho, onde foram submetidas à inoculação.

O inóculo foi preparado a partir de framboesas infectadas com $B$. cinerea e $R$. stolonifer, coletadas na própria área experimental. $\mathrm{O}$ crescimento das colônias foi obtido em placas de Petri, contendo meio batata-dextrose-ágar (BDA), mantidas a $22^{\circ} \mathrm{C}$. Para o preparo dos inóculos, as colônias foram lavadas com água destilada contendo $0,1 \%\left(\mathrm{v} \mathrm{v}^{-1}\right)$ de Tween 80 . A suspensão micelial obtida foi filtrada em gaze, e a concentração da suspensão conidial foi ajustada para $2 \times 10^{5}$ conídios $\mathrm{mL}^{-1}$, com uso de câmara de Neubauer. As frutas foram inoculadas individualmente por aspersão até o ponto de escorrimento, e acondicionadas em embalagens PET com 12 alvéolos, 1 hora após a inoculação. Em cada alvéolo, foi colocada uma única framboesa. A abertura das embalagens foi revestida por filme de policloreto de vinila (PVC). Foram utilizadas cinco repetições para cada um dos inóculos, tendose considerado cada embalagem com 12 framboesas como unidade amostral.

Para a avaliação da incidência de podridão, as frutas foram mantidas em câmara $\mathrm{BOD}$ a $12 \pm 0,5^{\circ} \mathrm{C}$ por sete dias e avaliadas após 1, 2, 3, 4 e 7 dias na primeira colheita; 3, 4, 5, 6 e 7 dias na segunda colheita; e 3, 5, 6 e 7 dias na terceira colheita. As avaliações realizadas aos 3 e 7 dias, comuns às três colheitas, foram utilizadas no cálculo da incidência média do tratamento. $\mathrm{O}$ cálculo da área abaixo da curva de progresso da incidência (AACPI) foi realizado para cada colheita, tendo-se utilizado todas as leituras de incidência disponíveis. Em seguida, foi calculada a AACPI média das três colheitas. O cálculo foi feito de acordo com Campbell \& Madden (1990), por meio da integração da curva de progresso da doença para cada parcela, com uso da fórmula:

$$
\text { AACPI }=\sum_{i=1}^{n}\left(\frac{\left(x_{i}+x_{i+1}\right)}{2}\right)\left(t_{i+1}-t_{i}\right),
$$

em que: $n$ é o número de avaliações, $x$ é a incidência da doença e $\left(t_{i+1}-t_{i}\right)$ é o intervalo entre as avaliações consecutivas.

Framboesas obtidas na primeira colheita (três dias após a realização dos tratamentos preventivos) foram avaliadas quanto aos seguintes atributos de qualidade: cor, teores de sólidos solúveis e acidez titulável, $\mathrm{pH}$, antocianinas, fenólicos totais e atividade antioxidante efetiva. A cor foi determinada com auxílio de espectrofotômetro CM-2500d, (Konica Minolta, São Paulo, SP), no sistema L*a*b*.

Os índices de cor foram calculados de acordo com as seguintes equações: chroma, $\mathrm{C}=\left(\mathrm{a}^{* 2}+\mathrm{b}^{* 2}\right)^{0,5}$; hue $\mathrm{h}^{\circ}=\tan ^{-1}\left(\mathrm{~b}^{*} / \mathrm{a}^{*}\right)$; e índice de cor, IC $=2.000 \times \mathrm{a}^{*} / \mathrm{L}^{*} \times$ $\left(\mathrm{a}^{* 2}+\mathrm{b}^{* 2}\right)^{0,5}$ (López Camelo \& Gómez, 2004). O teor de sólidos solúveis foi determinado por refratômetro PR-101, (Atago, Ribeirão Preto, SP), e os resultados foram expressos em ${ }^{\circ}$ Brix. A acidez titulável foi determinada por meio da diluição de $10 \mathrm{~g}$ de amostra homogeneizada em $100 \mathrm{~mL}$ de água destilada, seguida de titulação com solução de $\mathrm{NaOH} 0,1 \mathrm{~N}$ até pH 8,10 (Carvalho et al., 1990). Os valores foram expressos em percentual de ácido cítrico. $\mathrm{O} \mathrm{pH}$ foi determinado por potenciometria em amostra triturada e homogeneizada. A concentração de antocianinas foi determinada conforme Francis (1982) e expressa em miligramas de antocianinas por $100 \mathrm{~g}$ de fruta.

O extrato para determinação de compostos fenólicos totais e atividade antioxidante efetiva foi obtido conforme Larrauri et al. (1997). Já os fenólicos totais, foram determinados de acordo com Obanda et al. (1997). Em ambiente escuro, foram adicionados a $1 \mathrm{~mL}$ do extrato, $1 \mathrm{~mL}$ do reagente Folin-Ciocalteau (1:3), $2 \mathrm{~mL}$ de carbonato de sódio $20 \%$ e $2 \mathrm{~mL}$ de água destilada. A leitura foi realizada após $30 \mathrm{~min}$ em espectrofotômetro Evolution 60, (Thermo Scientific, Madison, WI, EUA) a $700 \mathrm{~nm}$. Os resultados foram expressos em miligramas de ácido gálico por $100 \mathrm{~g}$ de fruta. A determinação da atividade antioxidante efetiva foi realizada por meio da captura do radical livre ABTS, conforme Miller et al. (1993), com modificações (Rufino et al., 2010). Os resultados foram expressos em $\mu \mathrm{mol} \mathrm{L} \mathrm{L}^{-1}$ de trolox por grama de fruta. Foram utilizadas três repetições, tendo-se considerado como unidade amostral $100 \mathrm{~g}$ de framboesas contidas em recipiente PET.

Utilizou-se o delineamento experimental inteiramente casualizado com três e cinco repetições, respectivamente, para avaliação dos atributos de qualidade e de incidência de podridão. As medidas de incidência e de AACPI foram analisadas quanto à normalidade, pelo teste do qui-quadrado $\left(\chi^{2}\right)$, e quanto à homogeneidade das variâncias, pelo teste de Bartlett. Para o atendimento dos pressupostos de normalidade e homogeneidade, as medições de AACPI foram transformadas, quando necessário, para 
$\log (\mathrm{x}+0,5)$ e, em seguida, submetidas à análise de variância (ANOVA). A diferença entre as médias foi avaliada pelo teste de Tukey, a 5\% de probabilidade. Os dados de qualidade foram submetidos à ANOVA, e as médias foram comparadas pelo teste de Tukey, a 5\% de probabilidade. Para esses procedimentos, utilizouse o programa Statistica versão 6.0 (StatSoft, 2001).

\section{Resultados e Discussão}

A aplicação pré-colheita dos tratamentos influenciou o desenvolvimento das podridões de Botrytis e Rhizopus, e o tratamento com B. amyloliquefaciens foi o único que reduziu significativamente a AACPI em ambas as doenças (Tabela 1). As frutas tratadas com B. amyloliquefaciens e $S$. cerevisiae apresentaram as menores AACPI de podridão por Botrytis, que foram significativamente inferiores às observadas nas frutas-controle e nas que receberam o tratamento com quitosana. Quanto à podridão por Rhizopus, os tratamentos com C. pusillum e B. amyloliquefaciens

Tabela 1. Incidência de podridão por Botrytis cinerea e Rhizopus stolonifer em pós colheita de framboesa, aos três e sete dias após a inoculação (DAI) com os patógenos ${ }^{(1)}$, e área abaixo da curva de progresso da incidência (AACPI) ${ }^{(2)}$.

\begin{tabular}{|c|c|c|c|c|c|c|}
\hline \multirow[t]{2}{*}{ Tratamento } & \multicolumn{3}{|c|}{ Botrytis cinerea } & \multicolumn{3}{|c|}{ Rhizopus stolonifer } \\
\hline & $3 \mathrm{DAI}$ & $7 \mathrm{DAI}$ & AACPI & $3 \mathrm{DAI}$ & $7 \mathrm{DAI}$ & AACPI \\
\hline Controle & 25,0 & 98,1 & $227,2 b$ & 15,6 & 92,8 & $179,7 \mathrm{c}$ \\
\hline Quitosana & 23,1 & 91,0 & $223,7 b$ & 5,6 & 92,2 & $177,2 \mathrm{bc}$ \\
\hline Dióxido de cloro & 7,7 & 91,7 & $184,3 \mathrm{ab}$ & 11,7 & 94,4 & $186,4 \mathrm{c}$ \\
\hline Bacillus amyloliquefaciens & 7,7 & 83,3 & $150,0 \mathrm{a}$ & 11,9 & 79,2 & $142,9 \mathrm{ab}$ \\
\hline Curtobacterium pusillum & 9,0 & 92,9 & $188,5 \mathrm{ab}$ & 5,1 & 83,3 & $132,1 \mathrm{a}$ \\
\hline Saccharomyces cerevisiae & 8,3 & 91,7 & $176,6 \mathrm{a}$ & 9,6 & 87,8 & $163,8 \mathrm{abc}$ \\
\hline
\end{tabular}

${ }^{(1)}$ Valores médios de três colheitas sucessivas, realizadas aos 3, 7 e 14 dias após aplicação dos tratamentos. ${ }^{(2)}$ Médias seguidas de letras iguais não diferem entre si pelo teste de Tukey, a 5\% de probabilidade. proporcionaram menor AACPI, em comparação ao controle (Tabela 2).

Há relatos do uso de microrganismos no manejo de doenças foliares em outras culturas, como, por exemplo, o uso da levedura $S$. cerevisiae no progresso da mancha de Exserohilum turcicum em sorgo (Piccinin et al., 2005).

Em todos os tratamentos, a evolução das doenças atingiu mais de $79 \%$ das frutas avaliadas após sete dias da inoculação, o que indica que o método utilizado para inoculação e a agressividade dos isolados foram adequados para a avaliação dos tratamentos. $\mathrm{O}$ uso de agentes de controle biológico em pré-colheita pode ter duas abordagens: em aplicação única, próxima à colheita, ou em aplicações sucessivas durante todo o desenvolvimento do fruto (Janisiewicz \& Korsten, 2002). Em ambos os casos, o agente de controle biológico deve colonizar o fruto antes de o patógeno se instalar. Assim, as interações entre os microrganismos no filoplano tornam-se mais favoráveis ao agente de controle biológico e à inibição do agente patogênico.

Em relação aos atributos de qualidade, não foram observadas alterações quanto à coloração das framboesas controle e das submetidas aos diferentes tratamentos preventivos (Tabela 2). $\mathrm{O}$ valor médio de luminosidade da amostra variou entre 44,15 e 45,37, enquanto as coordenadas cromáticas a* e b* variaram de 13,48 a 14,96 e de 3,74 a 4,63, respectivamente.

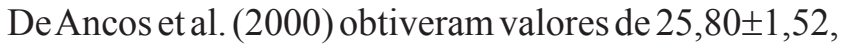
$34,98 \pm 0,58$ e $18,34 \pm 2,42$ para os componentes $L^{*}$, $a^{*}$ e $b^{*}$, respectivamente, na avaliação da cor de framboesas 'Heritage' cultivadas no Valle del Jerte, Cáceres, Espanha, e colhidas no estádio de maturação comercial. Os valores de $\mathrm{L}^{*}, \mathrm{a}^{*} \mathrm{e} \mathrm{b}^{*}$ obtidos no presente trabalho, para frutas controle, diferem dos observados por esses autores, o que pode ser atribuído,

Tabela 2. Atributos de qualidade de framboesas 'Heritage' submetidas a diferentes tratamentos em pré-colheita( ${ }^{(1)}$.

\begin{tabular}{|c|c|c|c|c|c|c|c|c|c|c|c|c|}
\hline Tratamento & $\mathrm{L}^{*}$ & $\mathrm{a}^{*}$ & $\mathrm{~b}^{*}$ & $\mathrm{C}^{*}$ & $\mathrm{~h}^{\circ}$ & IC & SS & AT & $\mathrm{pH}$ & ATC & CFT & $\mathrm{AAE}$ \\
\hline Controle & $45,37 \mathrm{a}$ & $14,54 \mathrm{a}$ & $4,34 \mathrm{a}$ & $15,17 \mathrm{a}$ & $16,57 \mathrm{a}$ & $42,26 a$ & $9,90 \mathrm{~b}$ & $2,45 \mathrm{a}$ & $2,70 \mathrm{~b}$ & $35,86 a$ & $191,89 a$ & $19,02 \mathrm{a}$ \\
\hline Quitosana & $45,15 \mathrm{a}$ & $14,83 a$ & $4,38 \mathrm{a}$ & $14,67 \mathrm{a}$ & $16,61 \mathrm{a}$ & $42,45 \mathrm{a}$ & $10,27 \mathrm{ab}$ & $2,30 \mathrm{a}$ & $2,68 b$ & $39,12 \mathrm{a}$ & $192,72 \mathrm{a}$ & $21,45 \mathrm{a}$ \\
\hline Dióxido de cloro & $44,15 \mathrm{a}$ & $14,05 \mathrm{a}$ & $4,20 \mathrm{a}$ & $15,46 \mathrm{a}$ & $16,45 \mathrm{a}$ & $43,46 \mathrm{a}$ & $10,67 \mathrm{ab}$ & $2,19 \mathrm{a}$ & $2,75 \mathrm{ab}$ & $34,88 \mathrm{a}$ & $168,56 \mathrm{a}$ & $20,05 \mathrm{a}$ \\
\hline Bacillus amyloliquefaciens & $45,15 \mathrm{a}$ & $13,87 \mathrm{a}$ & $3,99 \mathrm{a}$ & $14,43 a$ & $16,03 \mathrm{a}$ & $42,82 \mathrm{a}$ & $10,97 \mathrm{a}$ & $2,36 \mathrm{a}$ & $2,78 \mathrm{ab}$ & $36,61 \mathrm{a}$ & $188,64 \mathrm{a}$ & $22,13 a$ \\
\hline Curtobacterium pusillum & $44,92 \mathrm{a}$ & $14,96 \mathrm{a}$ & $4,63 \mathrm{a}$ & $13,99 \mathrm{a}$ & $15,48 \mathrm{a}$ & $42,92 \mathrm{a}$ & $10,23 \mathrm{ab}$ & $2,17 \mathrm{a}$ & $2,94 \mathrm{a}$ & $39,44 a$ & $200,30 \mathrm{a}$ & $19,09 \mathrm{a}$ \\
\hline Saccharomyces cerevisiae & $44,90 \mathrm{a}$ & $13,48 \mathrm{a}$ & $3,74 \mathrm{a}$ & $15,66 \mathrm{a}$ & $17,20 \mathrm{a}$ & $41,79 \mathrm{a}$ & $10,67 \mathrm{ab}$ & $2,38 \mathrm{a}$ & $3,00 \mathrm{ab}$ & $35,14 \mathrm{a}$ & $189,58 \mathrm{a}$ & $19,01 \mathrm{a}$ \\
\hline DMS (5\%) & 2,10 & 2,22 & 1,09 & 2,42 & 2,01 & 2,25 & 0,80 & 0,44 & 0,30 & 9,05 & 7,50 & 6,56 \\
\hline CV (\%) & 1,70 & 5,67 & 9,43 & 5,92 & 4,47 & 1,92 & 2,78 & 6,90 & 3,88 & 8,95 & 5,73 & 11,87 \\
\hline
\end{tabular}

${ }^{(1)}$ Médias seguidas de letras iguais, nas colunas, não diferem entre si pelo teste de Tukey, a 5\% de probabilidade. L*, luminosidade; a* e b*, coordenadas cromáticas; $\mathrm{C}^{*}$, cromaticidade; $\mathrm{h}^{\circ}$, ângulo hue; IC, índice de cor; SS, sólidos solúveis ( ${ }^{\circ}$ Brix); AT, acidez titulável (\% de ácido cítrico); ATC, antocianinas (mg por $100 \mathrm{~g}$ ); CFT, compostos fenólicos totais (mg por $100 \mathrm{~g}$ de fruta); AAE, atividade antioxidante efetiva ( $\mu \mathrm{mol} \mathrm{L}^{-1}$ de trolox). 
principalmente, ao estádio de maturação na colheita. A cromaticidade da amostra, representada pelo valor $C^{*}$, apresentou variação de 13,99 a 15,66. O ângulo de tonalidade, representado pelo valor $\mathrm{h}^{\circ}$, variou de 15,48 a 17,20. O ângulo de tonalidade inicia-se no eixo $+a^{*} \mathrm{e}$ é dado em graus, em que: $+a^{*}$ corresponde ao vermelho $\left(0^{\circ}\right),+b^{*}$ ao amarelo $\left(90^{\circ}\right),-a^{*}$ ao verde $\left(180^{\circ}\right)$ e $-b^{*}$ ao azul $\left(270^{\circ}\right)$. De modo semelhante, o índice de cor, cujos valores mais elevados indicam maior intensidade da coloração vermelha, variou de 41,79 a 43,46, sem apresentar diferença entre os tratamentos.

Frutas submetidas ao tratamento com B. amyloliquefaciens apresentaram teor de sólidos solúveis superior ao das frutas controle, ao passo que frutas submetidas aos demais tratamentos apresentaram teores intermediários. Os valores de $\mathrm{pH}$ observados nas framboesas tratadas com C. pusillum foram superiores aos obtidos nas frutas controle e nas tratadas com quitosana, sem diferir, no entanto, das frutas tratadas com dióxido de cloro, B. amyloliquefaciens e $S$. cerevisiae. Não houve diferença significativa entre os tratamentos quanto à acidez titulável. Como os atributos de qualidade normalmente estão relacionados ao estádio de maturação das frutas, os tratamentos podem ter interferido no processo de amadurecimento, fase final do desenvolvimento, quando as frutas sofrem uma série de alterações físicas, químicas e sensoriais que as tornam aptas para o consumo.

Não foi observada diferença entre os tratamentos quanto ao teor de antocianinas, compostos fenólicos totais e atividade antioxidante efetiva das frutas. Os teores de antocianinas observados estão de acordo com Howard \& Hager (2007), que obtiveram valores entre 19 e $80 \mathrm{mg}$ de cianidina 3-glucosídeo por $100 \mathrm{~g}$ de polpa, em framboesas vermelhas. Para a cultivar Heritage, foram relatados teores de antocianinas entre $22,4 \pm 0,2$ e 49,1 $\pm 7,8 \mathrm{mg}$ por $100 \mathrm{~g}$ (De Ancos et al., 2000; Pantelidis et al., 2007; Sariburun et al., 2010).

Quanto aos compostos fenólicos totais, os teores observados estão próximos aos menores valores obtidos por Howard \& Hager (2007) para framboesas vermelhas (192 a $512 \mathrm{mg}$ de ácido gálico por $100 \mathrm{~g}$ de frutos). No entanto, em relação à atividade antioxidante efetiva, os valores obtidos foram inferiores aos de Sariburun et al. (2010), que observaram 74,25 $\pm 1,47 \mu \mathrm{mol} \mathrm{g}{ }^{-1}$ de trolox na matéria fresca de framboesas 'Heritage'. A ampla variação entre os valores obtidos no presente trabalho e os encontrados na literatura pode ser decorrente das diferenças genéticas entre os materiais, das práticas culturais adotadas, das condições edafoclimáticas, do estádio de maturação na colheita, das diferentes condições de extração, dos procedimentos analíticos realizados, bem como dos padrões utilizados para a quantificação.

Quanto à aparência, verificou-se que a quitosana proporcionou a formação de uma película esbranquiçada sobre a fruta, que se soltou facilmente após a secagem do produto, o que inviabiliza sua utilização na concentração testada.

Os tratamentos com os microrganismos se mostraram promissores e são alternativas potenciais para o controle biológico de podridões pós-colheita de framboesas.

\section{Conclusão}

O uso de Bacillus amyloliquefaciens, Curtobacterium pusillum e Saccharomyces cerevisiae apresenta potencial para controle de podridões pós-colheita de framboesas e não interfere negativamente sobre os atributos de qualidade de framboesas 'Heritage'.

\section{Agradecimentos}

À Fundação de Amparo à Pesquisa do Estado do Rio Grande do Sul, pelo apoio financeiro; è A.D. Oceanum Indústria e Comércio Ltda., pela doação de amostra de quitosana para instalação dos experimentos.

\section{Referências}

AGROFIT. Agrofit [home page]. Disponível em: <http://extranet. agricultura.gov.br/agrofit_cons/principal_agrofit_cons $>$. Acesso em: 14 mar. 2011.

BOWER, C. Postharvest handling, storage, and treatment of fresh market berries. In: ZHAO, Y. Berry fruit: value-added products for health promotion. Boca Raton: CRC, 2007. p.262-288.

BRASIL. Ministério da Agricultura, Pecuária e Abastecimento. Secretaria de Defesa Agropecuária. Instrução normativa conjunta no 1 , de 23 de fevereiro de 2010. Diário Oficial [da] República Federativa do Brasil, n.36, 24 fev. 2010. Seção 1, p.19-21.

CAMPBELL, C.L.; MADDEN, L.V. Introduction to plant disease epidemiology. New York: John Wiley \& Sons, 1990. 532p.

CARVALHO, C.R.L.; MANTOVANI, D.M.B.; CARVALHO, P.R.N.; MORAES, R.M.M. Análises químicas de alimentos: manual técnico. Campinas: Instituto de Tecnologia de Alimentos, 1990. 121p. 
CARVALHO, V.L. de; CUNHA, R.L. da; CHALFUN, N.N.J.; MOURA, P.H.A. Alternativas de controle pós-colheita da podridão-parda e da podridão-mole em frutos de pessegueiro. Revista Brasileira de Fruticultura, v.31, p.78-83, 2009.

DE ANCOS, B.; GONZÁLEZ, E.M.; CANO, M.P. Ellagic acid, vitamin $\mathrm{C}$, and total phenolic contents and radical scavenging capacity affected by freezing and frozen storage in raspberry fruit. Journal of Agricultural and Food Chemistry, v.48, p.4565-4570, 2000 .

DEVLIEGHERE, F.; VERMEULEN, A.; DEBEVERE, J. Chitosan: antimicrobial activity, interactions with food components and applicability as a coating on fruit and vegetables. Food Microbiology, v.21, p.703-714, 2004.

EL GHAOUTH, A.; ARUL, J.; GRENIER, J.; ASSELIN, A. Glucanohydrolases and inhibitory activity to Botrytis cinerea in extracts from strawberry fruits. Canadian Journal of Plant Pathology, v.13, p.315-320, 1991.

FRANCIS, F.J. Analysis of anthocyanins. In: MARKAKIS, P. (Ed.). Anthocyanins as food colors. New York: Academic, 1982. p.181-207.

HAN, C.; LEDERER, C.; MCDANIEL, M.; ZHAO, Y. Sensory evaluation of fresh strawberries (Fragaria ananassa) coated with chitosan-based edible coatings. Journal of Food Science, v.70, p.172-178, 2005.

HOWARD, L.R.; HAGER, T.J. Berry fruit phytochemicals. In: ZHAO, Y. Berry fruit: value-added products for health promotion. Boca Raton: CRC Press, 2007. p.73-104.

JANISIEWICZ, W.J.; KORSTEN, L. Biological control of postharvest diseases of fruits. Annual Review of Phytopathology, V.40, p.411-441, 2002.

LARRAURI, J.A.; RUPÉREZ, P.; SAURA-CALIXTO, F. Effect of drying temperature on the stability of polyphenols and antioxidant activity of red grape pomace peels. Journal of Agricultural and Food Chemistry, v.45, p.1390-1393, 1997.

LÓPEZ CAMELO, A.F.; GÓMEZ, P.A. Comparison of color indexes for tomato ripening. Horticultura Brasileira, v.22, p.534-537, 2004.

MARI, M.; GUIZZARDI, M.; BRUNELLI, M.; FOLCHI, A. Postharvest biological control of grey mould (Botrytis cinerea Pers.: Fr.) on fresh-market tomatoes with Bacillus amyloliquefaciens. Crop Protection, v.15, p.699-705, 1996.

MAZARO, S.M.; DESCHAMPS, C.; MIO, L.L.M. de; BIASI, L.A.; GOUVEA, A. de; SAUTTER, C.K. Comportamento pós-colheita de frutos de morangueiro após a aplicação pré-colheita de quitosana e acibenzolar-S-metil-1. Revista Brasileira de Fruticultura, v.30, p.185-190, 2008.

MILLER, N.J.; RICE-EVANS, C.; DAVIES, M.J.; GOPINATHAN, V.; MILNER, A. A novel method for measuring antioxidant capacity and its application to monitoring the antioxidant status in premature neonates. Clinical Science, v.84, p.407-412, 1993.
MITCHAM, E. Quality of berries associated with preharvest and postharvest conditions. In: ZHAO, Y. Berry fruit: value-added products for health promotion. Boca Raton: CRC, 2007. p.207-228.

NUNES, M.C. do N. Raspberry. In: NUNES, M.C. do N. Color atlas of postharvest quality of fruits and vegetables. Ames: Blackwell Publishing, 2008. p.167-173.

OBANDA, M.; OWUOR, P.O; TAYLOR, S.J. Flavanol composition and caffeine content of green leaf as quality potential indicators of Kenyan black teas. Journal of the Science of Food and Agriculture, v.74, p.209-215, 1997.

PANTELIDIS, G.E.; VASILAKAKIS, M.; MANGANARIS, G.A.; DIAMANTIDIS, G.R. Antioxidant capacity, phenol, anthocyanin and ascorbic acid contents in raspberries, blackberries, red currants, gooseberries and Cornelian cherries. Food Chemistry, v.102, p.777-783, 2007.

PEN, L.T.; JIANG, Y.M. Effects of chitosan coating on shelf life and quality of fresh-cut Chinese water chestnut. Food Science and Technology, v.36, p.359-364, 2003.

PICCININ, E.; DI PIERO, R.M.; PASCHOLATI, S.F. Efeito de Saccharomyces cerevisiae na produtividade de sorgo e na severidade de doenças foliares no campo. Fitopatologia Brasileira, v.30, p.5-9, 2005.

REDDY, M.V.B.; BELKACEMI, K.; CORCUFF, R.; CASTAIGNE, F.; ARUL, J. Effect of pre-harvest chitosan sprays on post-harvest infection by Botrytis cinerea and quality of strawberry fruit. Postharvest Biology and Technology, v.20, p.39-51, 2000.

RUFINO, M. do S.M.; ALVES, R.E.; BRITO, E.S. de; PÉREZ-JIMÉNEZ, J.; SAURA-CALIXTO, F.; MANCINI-FILHO, J. Bioactive compounds and antioxidant capacities of 18 non-traditional tropical fruits from Brazil. Food Chemistry, v.121, p.996-1002, 2010.

SARIBURUN, E.; ŞAHIN, S.; DEMIR, C.; TÜRKBEN, C.; UYLASER, V. Phenolic content and antioxidant activity of raspberry and blackberry cultivars. Journal of Food Science, v.75, p.328-335, 2010.

SILVA, G.A. da; ALMEIDA, E.A. de. Production of yellow-green fluorescent pigment by Pseudomonas fluorescens. Brazilian Archives of Biology and Technology, v.49, p.411-419, 2006.

STATSOFT. STATISTICA: data analysis software system. Version 6. Tulsa: StatSoft, 2001. Available at: <www.statsoft. com>. Accessed on: 3 Oct. 2011.

ZHAO, Y. Berry fruit: value-added products for health promotion. Boca Raton: CRC, 2007. 430p.

ZOFFOLI, J.P.; LATORRE, B.A.; DAIRE, N.; VIERTEL, S. Efectividad del dióxido de cloro, en función de la concentración, $\mathrm{pH}$ y tiempo de exposición, en el control de Botrytis cinerea, Penicillium expansum y Rhizopus stolonifer. Ciencia e Investigación Agraria, v.32, p.181-188, 2005. 